\title{
Whether Intracranial Aneurysm Could Be Well Treated by Flow Diversion: A Comprehensive Meta-Analysis of Large-Sample Studies including Anterior and Posterior Circulation
}

\author{
Yingjin Wang $\mathbb{D}$, Changwei Yuan, Shengli Shen, Liqing Xu, and Hongzhou Duan $\mathbb{D}$ \\ Department of Neurosurgery, Peking University First Hospital, No. 8 Xishiku Street, Xicheng District, Beijing 100034, China \\ Correspondence should be addressed to Hongzhou Duan; duanhongzhou@126.com
}

Received 17 October 2020; Revised 12 February 2021; Accepted 26 February 2021; Published 10 March 2021

Academic Editor: Quintino Giorgio D'alessandris

Copyright (c) 2021 Yingjin Wang et al. This is an open access article distributed under the Creative Commons Attribution License, which permits unrestricted use, distribution, and reproduction in any medium, provided the original work is properly cited.

\begin{abstract}
Background. Flow diversion (FD) has become a widely adopted treatment method for intracranial aneurysms in the clinic, but a comprehensive meta-analysis of large-sample studies including anterior and posterior circulation is still lacking. Methods. The PubMed, Embase, Web of Science, and Cochrane databases were searched between January 1, 2008, and December 1, 2019. A random-effect model was used to calculate the efficacy and safety data as well as $95 \%$ confidence intervals (CIs). Results. The pooled sample size of all included studies was 6695 patients; the mean age was 55.5 years old, with a total of 7406 aneurysms. For efficacy, the complete occlusion rate in angiographic follow-up (AFU) at 6 months was $78 \%$ (95\% CI, 0.77, 0.80), and the AFU rate at 6-12 months was $90 \%$ (95\% CI, 0.88, 0.92). For safety, the hemorrhagic event rate was $2 \%$, the ischemic event rate was $5 \%$, and the mortality rate was $3 \%$. Conclusion. FD is an effective and safe treatment for intracranial aneurysm with high complete occlusion rate and acceptable complication rate.
\end{abstract}

\section{Background}

Over the past three decades, endovascular embolization with or without device assistance has been a widely adopted treatment method for intracranial aneurysms. However, a subgroup of lesions, including fusiform, wide-necked, and largeto-giant aneurysms, continues to present major challenges [1].

Fortunately, flow diversion (FD) is designed to provide sufficient metal coverage across the neck of the aneurysm to physiologically exclude the lesion from the circulation [2]. More importantly, flow diverters induce thrombosis into the aneurysmal sac while preserving physiological flow in the parent vessel and adjacent branches [3]. This excellent function is based on the special structure of a braided mesh cylinder composed of individual platinum and cobalt chromium microfilaments [4]. From the first case of a pipeline implantation [2] to the present, a large number of studies have reported the efficacy of flow diversion, and more advanced FD techniques have been designed and applied in clinical treatment, including silk FD and a flow-redirection endoluminal device $[5,6]$. Although a series of reviews and meta-analyses have been published over the past years [711], a comprehensive meta-analysis with a large sample data is still lacking.

The objective of this meta-analysis is to calculate a relatively more reliable result of the efficacy and safety of FD based on a large sample size and detailed data demonstration.

\section{Methods}

2.1. Search Strategy. We systematically searched the PubMed, Embase, Web of Science, and Cochrane databases between January 1, 2008 (the publication date of the first pipeline), and December 1, 2019, to identify all relevant articles on flow diversion in intracranial aneurysms. The following keywords were used in our searches: "Flow diversion" OR "Flow Diverter" OR "Flow-Diverting" OR "Pipeline" OR "PED" OR "Surpass" OR "Silk" OR "FRED"; "Intracranial Aneurysms" OR "Artery Aneurysms" OR "Cerebral Aneurysms" OR "Communicating Artery", with no language restrictions. Additionally, we searched the reference lists of the retrieved articles to further identify possibly eligible studies. The searches were 
performed independently by two investigators (Y.J.W. and C.W.Y), and any discrepancies were solved via discussion until a consensus was reached.

2.2. Selection Criteria and Quality Evaluation. Studies were included in the meta-analysis if they fulfilled the following criteria: (1) flow diversions were used for the treatment of intracranial aneurysms; (2) the outcome data included occlusion rates, complication rates, and follow-up time; and (3) sample size $>100$. Furthermore, studies were excluded if (1) they were case reports or case series; (2) they used a therapy method combined with FD or other endovascular materials, such as coils; (3) the studies did not report the occlusion rate in the article, sometimes the studies were retrospectively designed as a comparison between occluded and nonoccluded groups; and (4) the last angiographic follow-up was short term (several weeks or $<1$ month).

The quality evaluation for each study was performed using the guidelines from the Strengthening of Reporting of Observational studies in Epidemiology (STROBE) Statement, including a 22-item checklist (see Supplement 1). This study was planned and executed in accordance with the Preferred Reporting Items for Systematic Reviews and Meta-Analyses (PRISMA) guidelines [12].

2.3. Data Extraction. All data were independently extracted by two authors (Y.J.W. and C.W.Y.) using a customized data collection form. When necessary, the original authors were contacted for supplementary information. The following data were extracted: first author's name, publication year, country, patient number, aneurysm number, FD types, mean age, gender, design, clinical presentations, and aneurysm characteristics (size, location, and morphology). The outcome data included complete occlusion number/rate and complication events/rates.

2.4. Statistical Analysis and Outcome Assessment. To coordinate the outcome data from the included studies, we predefined the criterion of occlusion. We defined complete occlusion (CO, 100\% occlusion) as a valid therapeutic outcome. In addition, after data extraction, we predefined three groups in terms of postoperative angiographic follow-up (AFU): (1) AFU < 6 months, (2) AFU of 6 months, and (3) AFU between 6 and 12 months. The data from studies with a strict AFU of 6 months were sorted into group (2). In addition, the studies that did not have a preset AFU time period were sorted into group (1) or (3) according to their mean or last AFU time.

In the safety analysis, we examined several common complications: hemorrhagic events, ischemic events, and mortality rates. We defined hemorrhagic events (HEs) as subarachnoid hemorrhage (SAH), cerebral hemorrhage, intraventricular hemorrhage, and defined ischemic events (IEs) including any instances of cerebral embolization/thrombosis and transient ischemic attack (TIA). In terms of mortality, we did not integrate the primary data and merely pooled the records.

In this meta-analysis, a random-effect model was used to calculate the CO rate and all complications rates as well as
95\% confidence intervals (CIs). The statistical heterogeneity among the summary data was evaluated using the $I^{2}$ statistic $[13,14]$. We regarded that $I^{2}<40 \%$ indicated "heterogeneity might not be important" and $I^{2}>75 \%$ indicated "considerable heterogeneity" based on the suggestions provided by the Cochrane Handbook for Systemic Reviews of Interventions [15]. Heterogeneity was considered statistically significant if $p<0.05$.

To accurately evaluate the $\mathrm{CO}$ rate at the 6-month follow-up, we performed four subgroup analyses based on FD type, age, aneurysm dome size, and single/multicenter design.

\section{Results}

3.1. Search and Selection. From the primary search of the PubMed, Embase, Web of Science, and Cochrane databases and the manual search of reference lists, 932 potentially eligible records were identified. After screening the titles and abstracts, one hundred forty-eight articles were considered potentially eligible studies. After full-text screening, twentythree studies [16-38] met the inclusion criteria (Figure 1).

3.2. Patient and Aneurysm Characteristics. The pooled sample size of all included studies was 6695 patients with 7406 aneurysms. All the studies had a retrospective design and were published from 2012 to 2019. The mean patient age was 55.5 years old, with a total female rate of $76.8 \%$. The main clinical presentations of the patients were asymptomatic/incidental (42.7\%) and headache (25.4\%). Ten studies described preoperative modified Rankin Scale (mRS) scores, and thirteen studies listed mRS scores in the postoperative data. The detailed patient data are shown in Table 1 .

The characteristic data of the aneurysms are shown in Table 2. All studies were categorized into 4 regions (14 from North America, 5 from Europe, 2 from Asia, and 2 from Latin America). Regarding the sample sources, 17 studies were from multicenter sources, and six were from singlecenter sources. The mean size of the included aneurysms was $8.3 \pm 4.5 \mathrm{~mm}$. Subgroup analysis was performed by dome and neck lengths. The location of the aneurysms was listed by anterior circulation and posterior circulation. The aneurysms from the internal carotid, ophthalmic, cavernous, and clinoid arteries accounted for the main proportion. In terms of morphology, saccular and fusiform were the major sources. The basic characteristic data are shown in Supplement 2 .

3.3. Efficacy: Complete Occlusion Rate. The postoperative occlusion data in all follow-up periods were extracted in each study. The total complete occlusion rate was pooled from the maximum sample number of the AFU. From the results of the different follow-up periods, the complete occlusion rate for an $\mathrm{AFU}<6$ months was $68 \%$ (95\% CI, 0.65, 0.72), the complete occlusion rate for an AFU of 6 months was $78 \%$ (95\% CI, 0.77, 0.80), and the complete occlusion rate for an AFU of 6-12 months was $90 \%$ (95\% CI, 0.88, 0.92).

To identify the relationship between the results and some factors (FD type, age, dome size, and data source), a subgroup 

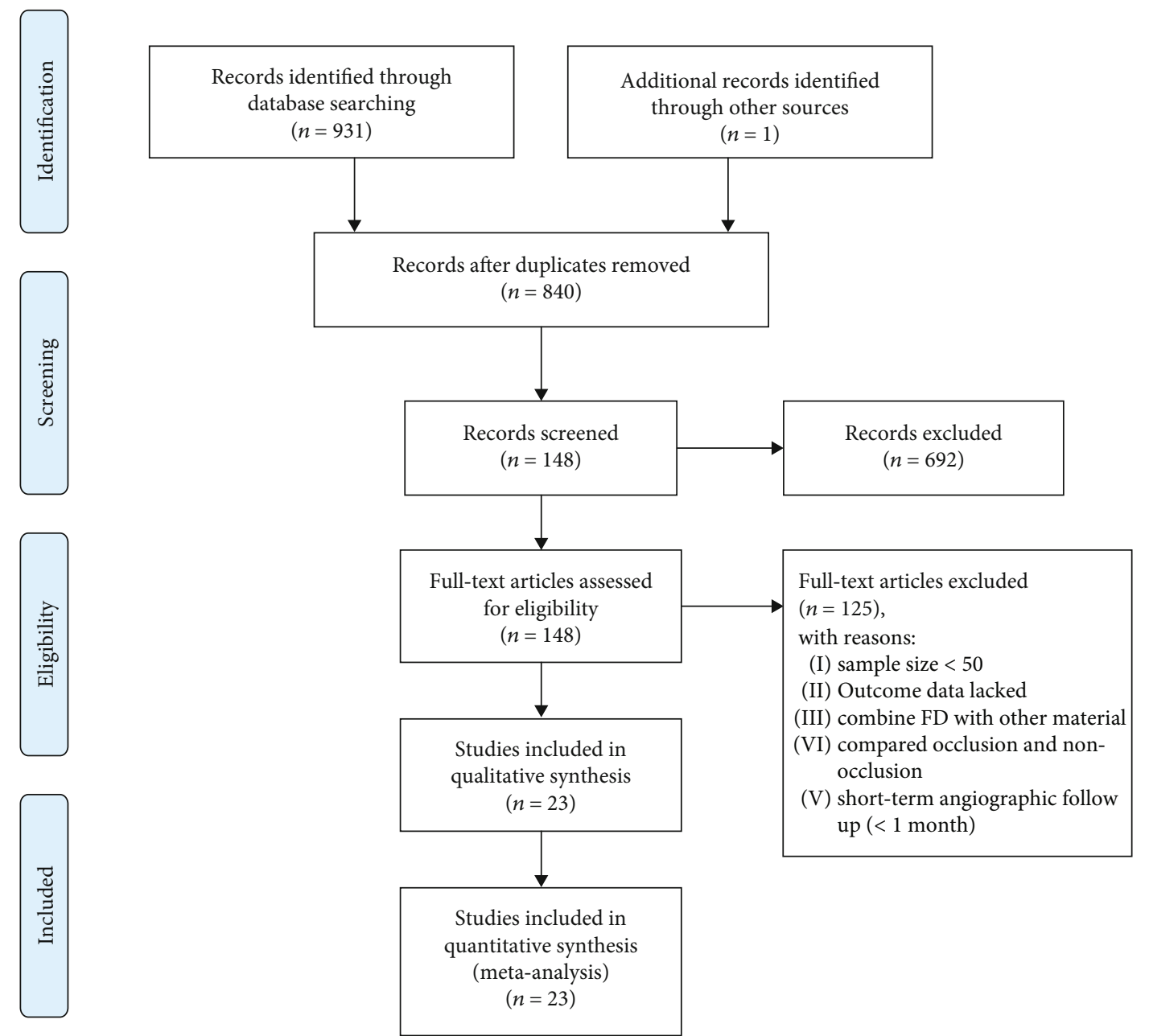

FIgURe 1: PRISMA flow chart of included eligible studies.

TABLE 1: Basic characteristics of patients.

\begin{tabular}{|c|c|c|c|}
\hline & Mean (SD) & $N$ of studies & $N$ of patients \\
\hline Age (year) & $55.5(13.4)$ & 23 & 6695 \\
\hline \multicolumn{2}{|l|}{ Female } & 23 & $5031(75.1 \%)$ \\
\hline \multicolumn{2}{|c|}{ Clinical presentation } & 13 & 1168 \\
\hline \multicolumn{2}{|c|}{ Asymptomatic/incidental } & $12 / 13$ & $872(74.7 \%)$ \\
\hline \multicolumn{2}{|c|}{ Headache } & $11 / 13$ & $517(44.3 \%)$ \\
\hline \multicolumn{2}{|c|}{ Hemorrhagic lesion } & $11 / 13$ & $203(17.3 \%)$ \\
\hline \multicolumn{2}{|c|}{ Ischemic lesion } & $5 / 13$ & $54(4.6 \%)$ \\
\hline \multicolumn{2}{|c|}{ Visual change } & $5 / 13$ & $78(6.7 \%)$ \\
\hline \multicolumn{2}{|c|}{ Other cranial nerve } & $6 / 13$ & $166(14.2 \%)$ \\
\hline \multicolumn{2}{|c|}{ Recurring } & $4 / 13$ & $150(12.8 \%)$ \\
\hline \multicolumn{2}{|c|}{ Preoperative mRS } & 10 & 2698 \\
\hline \multicolumn{2}{|c|}{ mRS 0-2 } & $10 / 10$ & $2591(96.0 \%)$ \\
\hline \multicolumn{2}{|c|}{ mRS 3-5 } & $10 / 10$ & $107(4.0 \%)$ \\
\hline \multicolumn{2}{|c|}{ Postoperative mRS } & 13 & 2449 \\
\hline \multicolumn{2}{|c|}{ mRS 0-2 } & $13 / 13$ & $2283(93.2 \%)$ \\
\hline \multicolumn{2}{|l|}{ mRS 3-5 } & $13 / 13$ & $166(6.8 \%)$ \\
\hline
\end{tabular}

Abbreviations: mRS: modified Rankin Scale. analysis was performed. The detailed data are shown in Table 3.

3.4. Safety: Complication Rate. Data on three postoperative outcomes (HEs, IEs, and mortality) were pooled to evaluate the safety of FD. As shown in Figure 2, the pooled HE rate was $2 \%(95 \% \mathrm{CI}, 0.02,0.03)$, the IE rate was $5 \%(95 \% \mathrm{CI}$, $0.04,0.06)$, and the mortality rate was $3 \%(95 \% \mathrm{CI}, 0.02$, $0.04)$.

3.5. Heterogeneity. For occlusion data, the total heterogeneity was negligible $\left(I^{2}=0.0 \%, p=0.567 ; I^{2}=9.7 \%, p=0.341 ; I^{2}\right.$ $=34.4 \%, p=0.133$, respectively). In terms of the safety outcome, the heterogeneity was moderately high for the HE and IE rates $\left(I^{2}=72.3 \%, p \leq 0.001 ; I^{2}=72.1 \%, p \leq 0.001\right.$, respectively). However, for mortality, the heterogeneity was fairly low $\left(I^{2}=0.0 \%, p=0.844\right)$.

\section{Discussion}

This is the first meta-analysis with the largest sample size, in terms of the efficacy and safety of FD, and all the included studies contained at least 100 patients. 
TABLE 2: Basic characteristics of aneurysms.

\begin{tabular}{|c|c|c|}
\hline Type & $N$ of studies & $N(\%)$ of aneurysms \\
\hline North America & 14 & $5210(70.3 \%)$ \\
\hline Europe & 5 & $1275(17.2 \%)$ \\
\hline Asia & 2 & $471(6.4 \%)$ \\
\hline Latin America & 2 & $450(6.1 \%)$ \\
\hline Single & 6 & $977(13.2 \%)$ \\
\hline Multi & 17 & $6429(86.8 \%)$ \\
\hline \multicolumn{3}{|l|}{ Aneurysm } \\
\hline Size (mean, mm) $8.3 \pm 4.5$ & 23 & 7406 \\
\hline Mean dome $<10 \mathrm{~mm}$ & 15 & $4440(60.0 \%)$ \\
\hline Mean dome $>10 \mathrm{~mm}$ & 8 & $2966(40.0 \%)$ \\
\hline Mean neck $<4 \mathrm{~mm}$ & 2 & $564(7.6 \%)$ \\
\hline Mean neck $>4 \mathrm{~mm}$ & 9 & $3015(40.7 \%)$ \\
\hline Unknown neck length & 12 & 3827 (51.7\%) \\
\hline \multicolumn{3}{|l|}{ Location } \\
\hline Anterior circulation & 21 & $5489(74.1 \%)$ \\
\hline ICA & 4 & $2169(29.3 \%)$ \\
\hline Ophthalmic & 14 & $1904(25.7 \%)$ \\
\hline Cavernous & 16 & $699(9.4 \%)$ \\
\hline Clinoid & 8 & $466(6.3 \%)$ \\
\hline MCA & 9 & $191(2.6 \%)$ \\
\hline ACA/ACoA & 4 & $60(0.8 \%)$ \\
\hline Posterior circulation & 12 & $461(6.2 \%)$ \\
\hline Vertebral & 5 & $142(1.9 \%)$ \\
\hline Basilar & 3 & $133(1.8 \%)$ \\
\hline PCA/PCoA & 7 & $186(2.5 \%)$ \\
\hline Unknown/other location & - & $1456(19.7 \%)$ \\
\hline Morphology & 15 & $5085(68.7 \%)$ \\
\hline Saccular & 15 & $4129(55.8 \%)$ \\
\hline Fusiform & 15 & $754(10.2 \%)$ \\
\hline Dissection & 8 & $160(2.2 \%)$ \\
\hline Blister & 5 & $42(0.6 \%)$ \\
\hline Unknown morphology & 8 & $2321(31.3 \%)$ \\
\hline
\end{tabular}

4.1. Population and Aneurysms. A total of 6695 patients with 7406 aneurysms were included in this meta-analysis. We described the extracted data according to category, including region, clinical presentations, data source design, and aneurysm location/size/morphology. Most patients were identified via medical examinations or headaches. The aneurysms were mainly located in the anterior circulation, in which lesions in the ICA and ophthalmic artery accounted for the main proportion. In terms of morphology, saccular and fusiform structures were more common. Based on a large number of aneurysms, the demonstration of epidemiological features could be more robust and reliable.

To review the published literature in the past decade, Arrese et al. [4] and Brinjikji et al. [8] performed relatively early meta-analyses in 2013, targeting intracranial aneurysms from 897 and 1451 patients, respectively. Subsequently, in the past three years, Cagnazzo et al. [39-42] and Sorenson et al. [43] performed their updated meta-analysis, illustrating detailed outcomes according to aneurysm location from the ACoA, MCA, PCoA, etc. For the recent meta-analysis, they included data with specific locations or morphologies so that their conclusions explained the specific problem and provided detailed evidence, while the sample size of some of their included studies was $<10[40,44]$. Including too many smallsample studies might result in a nonnegligible fluctuation of the results, just as the complete occlusion rate calculated by Kiyofuji et al. [44] was 52\% (29-76\%), while the record from Cagnazzo et al. [45] was 85.3\% (78.2-92.4\%). For unruptured nonsaccular intracranial aneurysms of the posterior circulation, the two studies also showed different outcomes. We consider that the pooled results of large sample can weaken the influence of operator's technique, case selection, and operation mode on the results and make the analysis results more objective and reliable.

4.2. Occlusion Rate. The evaluation of complete occlusion rate relied on different angiographic follow-up (AFU) periods. It was clear that longer AFU indicated higher occlusion rates. In our study, we strictly pooled the studies with a regular AFU of 6 months, in which the low heterogeneity will guarantee the reliability of the results, and the result showed that the accurate occlusion rate was 78\% (75-82\%) at the 6month AFU. Similarly, Brinjikji et al. [8] found a complete occlusion rate (mean AFU of 6 months) of 76\% (70-81\%), which was consistent with ours. In addition, we separately pooled the data of $<6$ months' and 6-12 months' group, and the CO rate was $68 \%(65-72 \%)$ and $90 \%(88-92 \%)$, respectively. The higher occlusion rate with time will increase the confidence of clinical use of FD in the future.

The correlation between occlusion rate and dome size was reported in some included studies. It showed that the complete occlusion rate of large $(>15 \mathrm{~mm})$ or giant-sized aneurysms $(>25 \mathrm{~mm})$ was lower than that of small aneurysms $(<7$ or $<10 \mathrm{~mm})$. The previous reviews $[8,44]$ also reported similar results to support this trend.

4.3. Complications. In this study, we integrated the records with subarachnoid or cerebral or ventricular hemorrhage as hemorrhage events and integrated the records with cerebral embolization/thrombosis, TIA, etc. as ischemic events. Compared with the HE rate, the IE rate was marginally higher (5\% vs. $2 \%$ ). The mortality rate was approximately $3 \%$ due to all causes.

Sorenson et al. [43] pooled the periprocedural complication rates of anterior cerebral artery and anterior communicating artery, showing ischemic stroke, hemorrhagic stroke, and mortality with records of $3 \%, 5 \%$, and $2 \%$, respectively. The result was consistent with ours, while our mortality rate was a little higher. In 2018, Cagnazzo et al. reported that the complication rate of posterior circulation was higher than that of anterior circulation ( $27 \%$ vs. $11.7 \%)$. In our metaanalysis, both anterior and posterior circulation aneurysms were included, and the slightly higher mortality might be 
explained by this. Furthermore, the $\mathrm{HE}$ and IE rates were consistent with most of the included studies and some of the published reviews, which confirmed that the application of FD in the treatment of aneurysm was relatively safe.

4.4. Quality Evaluation. Nonrandomized and retrospective studies are commonly considered to have low-level evidence, such as the included studies in this meta-analysis. However, most of the included studies had a multicenter design, and the sample size of each study was greater than 100. Therefore, we consider that most of the included studies could meet the medium level of evidence.

4.5. Strengths and Limitations. To our knowledge, this study might be the first meta-analysis of flow diversion with the largest sample size, as in each study the sample size was over 100. There are many advantages in our study. Firstly, based on the large sample size, we demonstrate detailed characteristic data of populations and aneurysms. Secondly, we evaluated both the efficacy and safety of FD with detailed and sufficient outcome data. Thirdly, the heterogeneity in the results of occlusion rate was low, which indicates the validity and reliability of the efficacy. Furthermore, PRISMA guidelines were followed to improve the quality of the present analysis and findings reported [12]. Therefore, the pooled occlusion rate in our study is considered to be reliable and accurate, based on the large sample sizes and markedly low heterogeneity.

Regarding the limitations, firstly and most importantly, the analysis model was a single-arm design without a parallel control group. Due to this limitation, this study was a descriptive analysis that merely described the efficacy and safety of FD rather than making a comparison. Secondly, although the detailed characteristics of the patients and aneurysms were listed, no detailed data could be analyzed with more subgroups (such as aneurysm location/morphology and clinical presentations). We excluded $<100$ sample studies, meanwhile losing more individual data published in case series. While Cagnazzo et al. [40-42, 45] and Sorenson et al. [43] have made up for this deficiency, they included studies with individual data so that the specific subgroup result could be supported. Finally, the heterogeneity in the HE and IE results was still nonnegligible, even though the results were consistent with most multicenter studies.

\section{Conclusion}

Based on the analysis with a large sample size and low heterogeneity, FD might be an effective and safe treatment for intracranial aneurysm with high complete occlusion rate and acceptable complication rate.
Abbreviations
ACA: Anterior cerebral artery
ACoA: Anterior communicating artery
ICA: Internal carotid artery
MCA: Middle cerebral artery
PCA: Posterior cerebral artery
PCoA: Posterior communicating artery

AFU: Angiographic follow-up

CO: Complete occlusion

FD: $\quad$ Flow diversion

PED: $\quad$ Pipeline embolization device

95\% CI: $95 \%$ confidence interval

mRS: Modified Rankin Scale

HEs: Hemorrhagic events

IEs: Ischemic events

\section{Data Availability}

The relevant data are available through the corresponding author upon reasonable request.

\section{Conflicts of Interest}

The authors declare that they have no competing interests.

\section{Authors' Contributions}

Author YJW and author CWY contributed to the data extraction and analysis. Author YJW and corresponding author HZD contributed to the foundation and manuscript checking. Author LQX and author SLS contributed to the manuscript revision. All authors agreed the order and declare no conflicts.

\section{Acknowledgments}

This study was supported by the Chinese National Nature Science Foundation (grant number 81541119), Peking University First Hospital Cross Clinical Study Foundation, and Youth Clinical Research Project of Peking University First Hospital (\#2019CR02).

\section{Supplementary Materials}

Supplement 1: STROBE scale assessment of all included studies. Supplement 2: basic characteristic of included studies. (Supplementary Materials)

\section{References}

[1] G. K. Leung, A. C. Tsang, and W. M. Lui, "Pipeline embolization device for intracranial aneurysm: a systematic review," Clinical Neuroradiology, vol. 22, no. 4, pp. 295-303, 2012.

[2] D. Fiorella, H. H. Woo, F. C. Albuquerque, and P. K. Nelson, "Definitive reconstruction of circumferential, fusiform intracranial aneurysms with the pipeline embolization device," Neurosurgery, vol. 62, no. 5, pp. 1115-1121, 2008, discussion 1120-1111.

[3] P. I. D'Urso, G. Lanzino, H. J. Cloft, and D. F. Kallmes, "Flow diversion for intracranial aneurysms: a review," Stroke, vol. 42, no. 8, pp. 2363-2368, 2011.

[4] I. Arrese, R. Sarabia, R. Pintado, and M. Delgado-Rodriguez, "Flow-diverter devices for intracranial aneurysms: systematic review and meta-analysis," Neurosurgery, vol. 73, no. 2, pp. 193-200, 2013, discussion 199-200.

[5] M. A. Mohlenbruch, C. Herweh, L. Jestaedt et al., "The FRED flow-diverter stent for intracranial aneurysms: clinical study to 
assess safety and efficacy," American Journal of Neuroradiology, vol. 36, no. 6, pp. 1155-1161, 2015.

[6] M. J. Binning, S. K. Natarajan, K. R. Bulsara, A. H. Siddiqui, L. N. Hopkins, and E. I. Levy, "SILK flow-diverting device for intracranial aneurysms," World Neurosurgery, vol. 76, no. 5, pp. 477.e1-477.e6, 2011.

[7] G. S. Sfyroeras, I. Dalainas, T. G. Giannakopoulos, K. Antonopoulos, J. D. Kakisis, and C. D. Liapis, "Flow-diverting stents for the treatment of arterial aneurysms," Journal of Vascular Surgery, vol. 56, no. 3, pp. 839-846, 2012.

[8] W. Brinjikji, M. H. Murad, G. Lanzino, H. J. Cloft, and D. F. Kallmes, "Endovascular treatment of intracranial aneurysms with flow diverters: a meta-analysis," Stroke, vol. 44, no. 2, pp. 442-447, 2013.

[9] S. B. Murthy, S. Shah, C. P. Venkatasubba Rao, E. M. Bershad, and J. I. Suarez, "Treatment of unruptured intracranial aneurysms with the pipeline embolization device," Journal of Clinical Neuroscience, vol. 21, no. 1, pp. 6-11, 2014.

[10] T. P. Madaelil, C. J. Moran, D. T. Cross 3rd, and A. P. Kansagra, "Flow diversion in ruptured intracranial aneurysms: a meta-analysis," American Journal of Neuroradiology, vol. 38, no. 3, pp. 590-595, 2017.

[11] F. Cagnazzo, P.-H. Lefevre, D. Mantilla et al., "Patency of the supraclinoid internal carotid artery branches after flow- diversion treatment. A meta-analysis," Journal of Neuroradiology, vol. 45, no. 2, pp. 100-114, 2018.

[12] D. Moher, A. Liberati, J. Tetzlaff, D. G. Altman, and for the PRISMA Group, "Preferred reporting items for systematic reviews and meta-analyses: the PRISMA statement," BMJ, vol. 339, no. jul21 1, article b2535, 2009.

[13] J. P. T. Higgins and S. G. Thompson, "Quantifying heterogeneity in a meta-analysis," Statistics in Medicine, vol. 21, no. 11, pp. 1539-1558, 2002.

[14] J. J. Deeks, D. G. Altman, and M. J. Bradburn, Statistical methods for examining heterogeneity and combining results from several studies in meta-analysis, Systematic Reviews in Health Care, 2008.

[15] J J J R S M Shuster, Review: Cochrane Handbook for Systematic Reviews for Interventions, Version 5.1.0, J. P. T. Higgins and S. Green, Eds., vol. 2, pp. 126-130, 2011.

[16] F. Briganti, M. Napoli, F. Tortora et al., "Italian multicenter experience with flow-diverter devices for intracranial unruptured aneurysm treatment with periprocedural complications-a retrospective data analysis," Neuroradiology, vol. 54, no. 10, pp. 1145-1152, 2012.

[17] I. Saatci, K. Yavuz, C. Ozer, S. Geyik, and H. S. Cekirge, "Treatment of intracranial aneurysms using the pipeline flowdiverter embolization device: a single-center experience with long-term follow-up results," American Journal of Neuroradiology, vol. 33, no. 8, pp. 1436-1446, 2012.

[18] T. Becske, D. F. Kallmes, I. Saatci et al., "Pipeline for uncoilable or failed aneurysms: results from a multicenter clinical trial," Radiology, vol. 267, no. 3, pp. 858-868, 2013.

[19] M. Piano, L. Valvassori, L. Quilici, G. Pero, and E. Boccardi, "Midterm and long-term follow-up of cerebral aneurysms treated with flow diverter devices: a single-center experience," Journal of Neurosurgery, vol. 118, no. 2, pp. 408-416, 2013.

[20] L. J. Kim, F. Tariq, M. Levitt et al., "Multimodality treatment of complex unruptured cavernous and paraclinoid aneurysms," Neurosurgery, vol. 74, no. 1, pp. 51-61, 2014.
[21] A. K. Wakhloo, P. Lylyk, J. de Vries et al., "Surpass flow diverter in the treatment of intracranial aneurysms: a prospective multicenter study," American Journal of Neuroradiology, vol. 36, no. 1, pp. 98-107, 2015.

[22] S. B. Yakovlev, S. R. Arustamyan, P. S. Dorokhov et al., "Endovascular treatment of large and giant intracranial aneurysms using flow-diverting stents," Voprosy neirokhirurgii imeni N.N. Burdenko, vol. 79, no. 4, pp. 19-27, 2015.

[23] O. Petr, W. Brinjikji, H. Cloft, D. F. Kallmes, and G. Lanzino, "Current trends and results of endovascular treatment of unruptured intracranial aneurysms at a single institution in the flow-diverter era," AJNR. American Journal of Neuroradiology, vol. 37, no. 6, pp. 1106-1113, 2016.

[24] W. Brinjikji, H. Cloft, S. Cekirge et al., "Lack of association between statin use and angiographic and clinical outcomes after pipeline embolization for intracranial aneurysms," American Journal of Neuroradiology, vol. 38, no. 4, pp. 753-758, 2017.

[25] J. E. Delgado Almandoz, Y. Kayan, A. Tenreiro et al., "Clinical and angiographic outcomes in patients with intracranial aneurysms treated with the pipeline embolization device: intraprocedural technical difficulties, major morbidity, and neurological mortality decrease significantly with increased operator experience in device deployment and patient management," Neuroradiology, vol. 59, no. 12, pp. 1291-1299, 2017.

[26] D. F. Kallmes, W. Brinjikji, S. Cekirge et al., "Safety and efficacy of the pipeline embolization device for treatment of intracranial aneurysms: a pooled analysis of 3 large studies," Journal of Neurosurgery, vol. 127, no. 4, pp. 775-780, 2017.

[27] M. B. Potts, M. Shapiro, D. W. Zumofen et al., "Parent vessel occlusion after pipeline embolization of cerebral aneurysms of the anterior circulation," Journal of Neurosurgery, vol. 127, no. 6, pp. 1333-1341, 2017.

[28] J. M. Pumar, A. Banguero, H. Cuellar et al., "Treatment of intracranial aneurysms with the SILK embolization device in a multicenter study. A retrospective data analysis," Neurosurgery, vol. 81, no. 4, pp. 595-601, 2017.

[29] N. Adeeb, C. J. Griessenauer, A. A. Dmytriw et al., "Risk of branch occlusion and ischemic complications with the pipeline embolization device in the treatment of posterior circulation aneurysms," American Journal of Neuroradiology, vol. 39, no. 7, pp. 1303-1309, 2018.

[30] L. B. C. Brasiliense, P. Aguilar-Salinas, D. K. Lopes et al., "Multicenter study of pipeline flex for intracranial aneurysms," Neurosurgery, vol. 84, no. 6, pp. E402-E409, 2019.

[31] G. Foa Torres, F. Roca, A. Noguera et al., "Silk flow-diverter stent for the treatment of complex intracranial aneurysms: a one-year follow-up multicenter study," Interventional neuroradiology: journal of peritherapeutic neuroradiology, surgical procedures and related neurosciences, vol. 24, no. 4, pp. 357$362,2018$.

[32] C. J. Griessenauer, A. J. Thomas, A. Enriquez-Marulanda et al., "Comparison of Pipeline Embolization Device and Flow ReDirection Endoluminal Device flow diverters for internal carotid artery aneurysms: a propensity score-matched cohort study," Neurosurgery, vol. 85, no. 2, pp. E249-E255, 2019.

[33] R. Gupta, C. S. Ogilvy, J. M. Moore et al., "Proposal of a followup imaging strategy following pipeline flow diversion treatment of intracranial aneurysms," Journal of Neurosurgery, vol. 131, no. 1, pp. 32-39, 2019.

[34] M. Killer-Oberpfalzer, N. Kocer, C. J. Griessenauer et al., "European multicenter study for the evaluation of a dual- 
layer flow-diverting stent for treatment of wide-neck intracranial aneurysms: the European flow-redirection intraluminal device study," American Journal of Neuroradiology, vol. 39, no. 5, pp. 841-847, 2018.

[35] M. A. Silva, A. P. See, P. Khandelwal et al., "Comparison of flow diversion with clipping and coiling for the treatment of paraclinoid aneurysms in 115 patients," Journal of Neurosurgery, vol. 130, no. 5, pp. 1505-1512, 2019.

[36] A. Sweid, E. Atallah, N. Herial et al., "Pipeline-assisted coiling versus pipeline in flow diversion treatment of intracranial aneurysms," Journal of Clinical Neuroscience, vol. 58, pp. 2024, 2018.

[37] F. P. Trivelato, M. T. Salles Rezende, A. C. Ulhoa, L. Henrique de Castro-Afonso, G. S. Nakiri, and D. G. Abud, "Occlusion rates of intracranial aneurysms treated with the pipeline embolization device: the role of branches arising from the sac," Journal of Neurosurgery, vol. 130, no. 2, pp. 543-549, 2019.

[38] A. A. Dmytriw, K. Phan, M. M. Salem et al., "The pipeline embolization device: changes in practice and reduction of complications in the treatment of anterior circulation aneurysms in a multicenter cohort," Neurosurgery, vol. 86, no. 2, pp. 266-271, 2020.

[39] F. Cagnazzo, D. T. di Carlo, M. Cappucci, P. H. Lefevre, V. Costalat, and P. Perrini, "Acutely ruptured intracranial aneurysms treated with flow-diverter stents: a systematic review and meta-analysis," American Journal of Neuroradiology, vol. 39, no. 9, pp. 1669-1675, 2018.

[40] F. Cagnazzo, N. Limbucci, S. Nappini et al., "Flow-diversion treatment of unruptured saccular anterior communicating artery aneurysms: a systematic review and meta-analysis," American Journal of Neuroradiology, vol. 40, no. 3, pp. 497502, 2019.

[41] F. Cagnazzo, P. Perrini, C. Dargazanli et al., "Treatment of unruptured distal anterior circulation aneurysms with flowdiverter stents: a meta-analysis," American Journal of Neuroradiology, vol. 40, no. 4, pp. 687-693, 2019.

[42] F. Cagnazzo, R. Ahmed, P. H. Lefevre et al., "Flow modification on the internal carotid artery bifurcation region and A1 segment after M1-internal carotid artery flow diverter deployment," Journal of NeuroInterventional Surgery, vol. 12, no. 12, pp. 1226-1230, 2020.

[43] T. J. Sorenson, V. Mendes Pereira, L. Rangel Castilla, C. A. Hilditch, P. J. Nicholson, and W. Brinjikji, "Treatment of anterior cerebral artery and anterior communicating artery aneurysms with flow-diversion devices: a systematic review and metaanalysis," Journal of Neurosurgical Sciences, vol. 64, no. 2, pp. 200-205, 2020.

[44] S. Kiyofuji, C. S. Graffeo, A. Perry et al., "Meta-analysis of treatment outcomes of posterior circulation non-saccular aneurysms by flow diverters," Journal of NeuroInterventional Surgery, vol. 10, no. 5, pp. 493-499, 2018.

[45] F. Cagnazzo, P. H. Lefevre, I. Derraz et al., "Flow-diversion treatment for unruptured nonsaccular intracranial aneurysms of the posterior and distal anterior circulation: a meta-analysis," American Journal of Neuroradiology, vol. 41, no. 1, pp. 134-139, 2020. 